\title{
Coefficient Matrix Decomposition Method and BIBO Stabilization of Stochastic Systems with Time Delays
}

\author{
Xia Zhou' ${ }^{1}$ and Shouming Zhong ${ }^{2}$ \\ ${ }^{1}$ School of Mathematics and Computational Science, Fuyang Teachers College, Fuyang, Anhui 236037, China \\ ${ }^{2}$ College of Mathematical Sciences, University of Electronic Science and Technology of China, Chengdu, Sichuan 611731, China \\ Correspondence should be addressed to Xia Zhou; zhouxia44185@163.com
}

Received 28 June 2013; Accepted 6 November 2013

Academic Editor: Nazim Idrisoglu Mahmudov

Copyright (c) $2013 \mathrm{X}$. Zhou and S. Zhong. This is an open access article distributed under the Creative Commons Attribution License, which permits unrestricted use, distribution, and reproduction in any medium, provided the original work is properly cited.

The mean square BIBO stabilization is investigated for the stochastic control systems with time delays and nonlinear perturbations. A class of suitable Lyapunov functional is constructed, combined with the descriptor model transformation and the decomposition technique of coefficient matrix; thus some novel delay-dependent mean square BIBO stabilization conditions are derived. These conditions are expressed in the forms of linear matrix inequalities (LMIs), whose feasibility can be easily checked by using Matlab LMI Toolbox. Finally, three numerical examples are given to demonstrate that the derived conditions are effective and much less conservative than those given in the literature.

\section{Introduction}

Because of the finite switching speed, memory effects, and so on, time delay is unavoidable in technology and nature, which commonly exists in various mechanical, chemical engineering, physical, biological, and economic systems. It can make the concerned control systems become of poor performance and unstable, which leads to the difficulty of hardware implementation of the control system. Thus, the stability of time-delay systems has been widely investigated. See [1-7] and some references therein. For systems with small delay, a model transformation technique is often used to transform the system with discrete delay into a system with distributed delay; the advantage of this transformation is to transform the original system to an equivalent descriptor from representation and additional dynamics in the systems will not be introduced. The delay-dependent stabilization criteria obtained by the coefficient matrix decomposition method are usually less conservative than some existing ones. Please refer to [3]. In recent years, bounded-input boundedoutput (BIBO) stabilization has been investigated by many researchers in order to track out the reference input signal in real world; see [5-22] and some references therein. In $[15,16]$, the sufficient condition for BIBO stabilization of control systems with no delays is proposed by the Biharitype inequality. In $[6,7]$, the BIBO stabilization of the systems without distributed time delays was investigated by employing the parameters technique and the Gronwall inequality. In [17-19], some BIBO stabilization criteria for a class of delayed control systems with nonlinear perturbations were established, based on Riccati equations, by constructing appropriate Lyapunov functions. In [20], the BIBO stabilization problem of a class of piecewise switched linear systems was further investigated.

However, up to now, these previous results have been assumed to be in deterministic systems, including continuous time deterministic systems and discrete time deterministic systems, but seldom in stochastic systems (see [21, 22]; in [21] Fu and Liao got several mean square BIBO stabilization criteria in terms of Razumikhin technique and comparison principle. In [22], Zhou and Zhong discussed the mean square BIBO stabilization of the stochastic delay system with nonlinear perturbations by auxiliary algebraic Riccati matrix equations). In practice, stochastic control systems are more applicable to problems that are environmentally noisy in nature or related to biological realities. Thus, the BIBO stabilization analysis problems for stochastic control case are necessary. 
Motivated by the previous discussions, this paper mainly aims to study the BIBO stabilization in mean square for the stochastic control systems with time delays and nonlinear perturbations. Based on the descriptor model transformation and the decomposition technique of coefficient matrix, some sufficient conditions guaranteeing BIBO stabilization in mean square are obtained. Finally, three numerical examples provided to demonstrate the derived conditions are valid and much less conservative than those given in the literature.

Notations. The notations are quite standard. Throughout this letter, $R^{n}$ and $R^{n \times m}$ denote, respectively, the $n$-dimensioned Euclidean space and the set of all $n \times m$ real matrices. The superscript " $T$ " denotes the transpose and the notation $X \geq Y$ (resp., $X>Y$ ) means that $X$ and $Y$ are symmetric matrices and that $X-Y$ is positive semidefinitive (respective positive definite). $\|\cdot\|$ is the Euclidean norm in $R^{n}$. I is the identity matrix with compatible dimension. $A$ is a matrix, denoted by $\|A\|$ as its operator norm; that is, $\|A\|=\sup \{\|A x\|$ : $\|x\|=1\}=\sqrt{\lambda_{\max }\left(A^{T} A\right)}$, where $\lambda_{\max }(A)$ (resp., $\left.\lambda_{\min }(A)\right)$ means the largest (resp., smallest) eigenvalue of $A$. Moreover, let $\left(\Omega, F,\left\{F_{t}\right\}_{t \geq 0}, P\right)$ be a complete probability space with a filtration $\left\{F_{t}\right\}_{t \geq 0}$ satisfying the usual conditions (i.e., the filtration contains all $P$-null sets and is right continuous). $\mathbb{E}\{\cdot\}$ stands for the mathematical expectation operator with respect to the given probability measure $P$. The asterisk * in a matrix is used to denote term that is induced by symmetry. Matrices, if not explicitly specified, are assumed to have compatible dimensions. Sometimes, the arguments of function will be omitted in the analysis when no confusion can arise.

\section{Problem Formulation and Preliminaries}

Consider the stochastic control system described by the following equation:

$$
\begin{aligned}
& d x(t)=\left[A x(t)+B x\left(t-\tau_{1}\right)\right. \\
& \left.+f\left(t, x(t), x\left(t-\tau_{1}\right)\right)+C u(t)\right] d t \\
& +\left[G x(t)+H x\left(t-\tau_{2}\right)\right] d \mathbf{w}(t), \quad t \geq t_{0} \geq 0, \\
& y(t)=D x(t) \\
& x(\theta)=\varphi(\theta) \in C_{\mathscr{F}_{0}}^{b}\left(\left[t_{0}-\tau, t_{0}\right] ; R^{n}\right), \quad \theta \in\left[t_{0}-\tau, t_{0}\right],
\end{aligned}
$$

where $x(t), u(t)$, and $y(t)$ are the state vector, control input, and control output of the system, respectively. $\tau_{1}>0, \tau_{2}>0$ are discrete time delays, and $\tau=\max \left\{\tau_{1}, \tau_{2}\right\} . A, B, C, D, G$, and $H$ are constant matrices with appropriate dimensional, $\mathbf{w}(t)=\left(\mathbf{w}_{1}(t), \mathbf{w}_{2}(t), \ldots, \mathbf{w}_{n}(t)\right)^{T}$ is an $n$-dimensional standard Brownian motion defined on a complete probability space $\left(\Omega, F,\left\{F_{t}\right\}_{t \geq 0}, P\right)$ with a natural filtration $\left\{F_{t}\right\}_{t \geq 0}$, and $f\left(t, x(t), x\left(t-\tau_{1}\right)\right) \in C\left(R^{+} \times R^{n} \times R^{n}, R^{n}\right)$ is the nonlinear vector-valued perturbation bounded in magnitude as

$$
\left\|f\left(t, x(t), x\left(t-\tau_{1}\right)\right)\right\|^{2} \leq \alpha_{1}\|x(t)\|^{2}+\alpha_{2}\left\|x\left(t-\tau_{1}\right)\right\|^{2},
$$

where $\alpha_{1}, \alpha_{2}$ are known positive constants.

To obtain the control law described by (1) and to track out the reference input of the system, we let the controller be in the form of

$$
u(t)=K x(t)+r(t)
$$

where $K$ is the feedback gain matrix and $r(t)$ is the reference inputs.

At the end of this section, let us introduce some important definitions and lemmas which will be used in the sequel.

Definition 1 (see [21]). A vector function $r(t)=\left(r_{1}(t)\right.$, $\left.r_{2}(t), \ldots, r_{n}(t)\right)^{T}$ is said to be an element of $L_{\infty}^{n}$, if $\|r\|_{\infty}=$ $\sup _{t \in\left[t_{0},+\infty\right)}\|r(t)\|<+\infty$, where $\|\cdot\|$ denotes the Euclid norm in $R^{n}$, or the norm of a matrix.

Definition 2 (see [21]). The nonlinear stochastic control system (1) is mean square BIBO stabilization, if one can construct controller (3) such that the output $y(t)$ satisfies

$$
\mathbb{E}\left(\|y(t)\|^{2}\right) \leq N_{1}+N_{2}\|r\|_{\infty}^{2},
$$

where $N_{1}, N_{2}$ are positive constants.

Definition 3 (see [21], $\mathscr{L}$ operator). Let Lyapunov functionals $V: C\left([-\tau, 0] ; R^{n}\right) \times R_{+} \rightarrow R$; its infinitesimal operator $\mathscr{L}$, acting on functional $V$, is defined by

$$
\mathscr{L} V\left(x_{t}, t\right)=\lim _{\Delta \rightarrow 0^{+}} \sup \frac{1}{\Delta}\left[\mathbb{E}\left(V\left(x_{t+\Delta}, t+\Delta\right)-V\left(x_{t}, t\right)\right)\right] .
$$

Lemma 4 (see [23]). For any constant symmetric matrix $M \in$ $R^{n \times n}, M=M^{T}>0$, scalar $r>0$, and vector function $g$ : $[0, r] \rightarrow R^{n}$, such that the integrations in the following are well defined, and then

$$
r \int_{0}^{r} g^{T}(s) M g(s) d s \geq\left[\int_{0}^{r} g(s) d s\right]^{T} M\left[\int_{0}^{r} g(s) d s\right] .
$$

Lemma 5 (see [24]). Let $x, y \in R^{n}$ and any $n \times n$ positivedefinite matrix $Q>0$. Then, one has

$$
2 x^{T} y \leq x^{T} Q^{-1} x+y^{T} Q y .
$$

\section{BIBO Stabilization for Nonlinear Stochastic Systems}

To derive delay-dependent mean square stabilization conditions, which include the information of the time delay $\tau_{1}$, one usually uses the fact

$$
x\left(t-\tau_{1}\right)=x(t)-\int_{t-\tau_{1}}^{t} \dot{x}(s) d s
$$


to transform the original system to a system with distributed delays. Let us decompose the coefficient matrix $B$ as $B=$ $B_{1}+B_{2}$, where $B_{1}, B_{2}$ are constant matrices. Then the original system (1) can be represented in the form of the descriptor system with discrete and distributed delays:

$$
\begin{gathered}
d\left[x(t)+B_{2} \int_{t-\tau_{1}}^{t} x(s) d s\right] \\
=\left[\left(A+B_{2}+C K\right) x(t)+B_{1} x\left(t-\tau_{1}\right)\right. \\
\left.+C r(t)+f\left(t, x(t), x\left(t-\tau_{1}\right)\right)\right] d t \\
+\left[G x(t)+H x\left(t-\tau_{2}\right)\right] d \mathbf{w}(t), \quad t \geq t_{0} \geq 0, \\
y(t)=D x(t), \\
x(\theta)=\varphi(\theta) \in C_{\mathscr{F}_{0}}^{b}\left(\left[t_{0}-\tau, t_{0}\right] ; R^{n}\right), \quad \theta \in\left[t_{0}-\tau, t_{0}\right] .
\end{gathered}
$$

Letting $D\left(x_{t}\right)$ be a new operator, we have

$$
D\left(x_{t}\right)=x(t)+B_{2} \int_{t-\tau_{1}}^{t} x(s) d s .
$$

To guarantee that the difference operator $D\left(x_{t}\right): C[-\tau, 0] \rightarrow$ $R^{n}$ given by (10) is stable, we assume the following [25]:

A1: let $\tau_{1}\left\|B_{2}\right\|<1$, where $\|\cdot\|$ is any matrix norm.

For the mean square BIBO stabilization of the system described by (9) and (3), we have the following results.

Theorem 6. For any given positive constants $\beta_{i}>0, i=$ $1,2,3,4,5$, the nonlinear stochastic control system (1) with the controller (3) is mean square BIBO stabilization, if there exist symmetric positive-definite matrices $P, R_{1}, R_{2}, R_{3}$, and $X$, such that

$$
\begin{aligned}
& \beta_{5} \lambda_{\text {min }}(P)-\beta_{1} \lambda_{\text {max }}\left(R_{1}\right)-\beta_{2} \lambda_{\text {max }}\left(R_{2}\right) \\
& \quad-\beta_{4}\left(\alpha_{1}+\alpha_{2}\right)-\tau_{1}^{2} \lambda_{\max }\left(\beta_{3} R_{3}+\beta_{5} B_{2}^{T} P B_{2}\right)>0,
\end{aligned}
$$

and the linear matrix inequality

$$
\widetilde{\Xi}=\left(\begin{array}{ccccc}
(1,1) & P B_{1} & G^{T} P H & (1,4) & P \\
* & (2,2) & 0 & 0 & 0 \\
* & * & (3,3) & -H^{T} P G B_{2} & 0 \\
* & * & * & (4,4) & 0 \\
* & * & * & * & -\beta_{4} I
\end{array}\right) \leq 0,
$$

with

$$
\begin{gathered}
(1,1)=P A+A^{T} P+P B_{2}+B_{2}^{T} P \\
+G^{T} P G-X-X^{T}+\beta_{5} P+P \\
(1,4)=-P A B_{2}-P B_{2}^{2}-G^{T} P G B_{2}+X B_{2}-\beta_{5} P B_{2}, \\
(2,2)=-\beta_{1} R_{1}, \\
(3,3)=H^{T} P H-\beta_{2} R_{2}, \\
(4,4)=B_{2}^{T} G^{T} P G B_{2}-\beta_{3} R_{3} .
\end{gathered}
$$

Proof. We define a Lyapunov functional $V\left(t, x_{t}\right)$ as

$$
V\left(t, x_{t}\right)=V_{1}\left(t, x_{t}\right)+V_{2}(t)+V_{3}(t)+V_{4}(t),
$$

where

$$
\begin{gathered}
V_{1}\left(t, x_{t}\right)=D^{T}\left(x_{t}\right) P D\left(x_{t}\right), \\
V_{2}(t)=\int_{t-\tau_{1}}^{t} x^{T}(s)\left(\beta_{1} R_{1}+\beta_{4} \alpha_{2}\right) x(s) d s, \\
V_{3}(t)=\beta_{2} \int_{t-\tau_{2}}^{t} x^{T}(s) R_{2} x(s) d s, \\
V_{4}(t)=\tau_{1} \int_{t-\tau_{1}}^{t}\left(s-t+\tau_{1}\right) x^{T}(s)\left(\beta_{3} R_{3}+\beta_{5} B_{2}^{T} P B_{2}\right) x(s) d s .
\end{gathered}
$$

Taking the operator $\mathscr{L}$ of $V_{1}\left(t, x_{t}\right)$ along the trajectory of system (1), we have

$$
\begin{aligned}
\mathscr{L} V_{1}\left(t, x_{t}\right)=D^{T}\left(x_{t}\right) P\left[\left(A+B_{2}+C K\right) x(t)+B_{1} x\left(t-\tau_{1}\right)\right. & \left.+f\left(t, x(t), x\left(t-\tau_{1}\right)\right)+C r(t)\right] \\
& +\left[\left(A+B_{2}+C K\right) x(t)+B_{1} x\left(t-\tau_{1}\right)+C r(t)\right. \\
& \left.+f\left(t, x(t), x\left(t-\tau_{1}\right)\right)\right]^{T} P D\left(x_{t}\right) \\
& +\frac{1}{2} \operatorname{trace}\left[\left(G x(t)+H x\left(t-\tau_{2}\right)\right)^{T}\right. \\
& \left.\quad \times 2 P\left(G x(t)+H x\left(t-\tau_{2}\right)\right)\right] \\
\leq & \xi(t)^{T} \widetilde{\Xi} \xi(t)+\beta_{1} x^{T}\left(t-\tau_{1}\right) R_{1} x\left(t-\tau_{1}\right) \\
& +D^{T}\left(x_{t}\right) P C r(t)+\beta_{2} x^{T}\left(t-\tau_{2}\right) R_{2} x\left(t-\tau_{2}\right) \\
& -\beta_{5} D^{T}\left(x_{t}\right) P D\left(x_{t}\right) \\
& +\beta_{3}\left(\int_{t-\tau_{1}}^{t} x(s) d s\right)^{T} R_{3}\left(\int_{t-\tau_{1}}^{t} x(s) d s\right) \\
& +r^{T}(t) C^{T} P D\left(x_{t}\right)+\beta_{4} f^{T}\left(t, x(t), x\left(t-\tau_{1}\right)\right) \\
& \times f\left(t, x(t), x\left(t-\tau_{1}\right)\right),
\end{aligned}
$$

where

$$
\begin{gathered}
\xi(t)^{T}=\left[D^{T}\left(x_{t}\right), x^{T}\left(t-\tau_{1}\right), x^{T}\left(t-\tau_{2}\right),\right. \\
\left.\quad\left(\int_{t-\tau_{1}}^{t} x(s) d s\right)^{T}, f^{T}\left(x, x(t), x\left(t-\tau_{1}\right)\right)\right], \\
\widetilde{\Xi}=\left(\begin{array}{ccccc}
(\widetilde{1}, \widetilde{1}) & P B_{1} & G^{T} P H & (\widetilde{1}, \widetilde{4}) & P \\
* & -\beta_{1} R_{1} & 0 & 0 & 0 \\
* & * & H^{T} P H-\beta_{2} R_{2} & -H^{T} P G B_{2} & 0 \\
* & * & * & B_{2}^{T} G^{T} P G B_{2}-\beta_{3} R_{3} & 0 \\
* & * & * & * & -\beta_{4} I
\end{array}\right) \leq 0,
\end{gathered}
$$


with

$$
\begin{gathered}
(\widetilde{1}, \widetilde{1})=P A+A^{T} P+P B_{2}+B_{2}^{T} P+G^{T} P G-X-X^{T}+\beta_{5} P, \\
(\widetilde{1}, \widetilde{4})=-P A B_{2}-P B_{2}^{2}-G^{T} P G B_{2}+X B_{2} .
\end{gathered}
$$

By Lemmas 4 and 5, (2), and Definition 1 we conclude that

$$
\begin{aligned}
\mathscr{L} V_{1} & \left(t, x_{t}\right) \\
\leq & \xi(t)^{T} \widetilde{\Xi} \xi(t)+x^{T}\left(t-\tau_{1}\right)\left(\beta_{1} R_{1}+\beta_{4} \alpha_{2}\right) x\left(t-\tau_{1}\right) \\
& +\beta_{2} x^{T}\left(t-\tau_{2}\right) R_{2} x\left(t-\tau_{2}\right) \\
& +\beta_{4} \alpha_{1}\|x(t)\|^{2}-\beta_{5} x^{T}(t) P x(t) \\
& +\tau_{1} \int_{t-\tau_{1}}^{t} x^{T}(s)\left(\beta_{3} R_{3}+\beta_{5} B_{2}^{T} P B_{2}\right) x(s) d s \\
& +D^{T}\left(x_{t}\right) P D\left(x_{t}\right) \\
& -\beta_{5} D^{T}\left(x_{t}\right) P B_{2} \int_{t-\tau_{1}}^{t} x^{T}(s) d s \\
& -\beta_{5} \int_{t-\tau_{1}}^{t} x^{T}(s) d s B_{2}^{T} P D\left(x_{t}\right) \\
& +\left\|C^{T} P C\right\|\|r\|_{\infty}^{2} .
\end{aligned}
$$

Taking the operator $\mathscr{L}$ of $V_{i}(t), i=2,3,4$, along the trajectory of system (1), we get

$$
\begin{gathered}
\mathscr{L} V_{2}(t)=x^{T}(t)\left(\beta_{1} R_{1}+\beta_{4} \alpha_{2}\right) x(t) \\
-x^{T}\left(t-\tau_{1}\right)\left(\beta_{1} R_{1}+\beta_{4} \alpha_{2}\right) x\left(t-\tau_{1}\right), \\
\mathscr{L} V_{3}(t)=\beta_{2} x^{T}(t) R_{2} x(t)-\beta_{2} x^{T}\left(t-\tau_{2}\right) R_{2} x\left(t-\tau_{2}\right), \\
\mathscr{L} V_{4}(t)=\tau_{1}^{2} x^{T}(t)\left(\beta_{3} R_{3}+\beta_{5} B_{2}^{T} P B_{2}\right) x(t) \\
-\tau_{1} \int_{t-\tau_{1}}^{t} x^{T}(s)\left(\beta_{3} R_{3}+\beta_{5} B_{2}^{T} P B_{2}\right) x(s) d s .
\end{gathered}
$$

Combining (20) with (22), we have

$$
\begin{aligned}
\mathscr{L} V\left(t, x_{t}\right) \leq & \xi(t)^{T} \widetilde{\Xi} \xi(t)-\beta_{5} x^{T}(t) P x(t) \\
& +x^{T}(t)\left(\beta_{1} R_{1}+\beta_{2} R_{2}\right) x(t) \\
& +x^{T}(t)\left(\beta_{4} \alpha_{1}+\beta_{4} \alpha_{2}\right) x(t)+\left\|C^{T} P C\right\|\|r\|_{\infty}^{2} \\
& +\tau_{1}^{2} x^{T}(t)\left(\beta_{3} R_{3}+\beta_{5} B_{2}^{T} P B_{2}\right) x(t)
\end{aligned}
$$

$$
\leq \xi(t)^{T} \widetilde{\Xi} \xi(t)
$$$$
\text { - }\left(\beta_{5} \lambda_{\text {min }}(P)-\beta_{1} \lambda_{\max }\left(R_{1}\right)\right.
$$$$
-\beta_{2} \lambda_{\max }\left(R_{2}\right)-\beta_{4}\left(\alpha_{1}+\alpha_{2}\right)
$$$$
\left.-\tau_{1}^{2} \lambda_{\max }\left(\beta_{3} R_{3}+\beta_{5} B_{2}^{T} P B_{2}\right)\right)\|x(t)\|^{2}
$$$$
+\left\|C^{T} P C\right\|\|r\|_{\infty}^{2} \text {. }
$$

Let $a=\beta_{5} \lambda_{\text {min }}(P)-\beta_{1} \lambda_{\text {max }}\left(R_{1}\right)-\beta_{2} \lambda_{\text {max }}\left(R_{2}\right)-\beta_{4}\left(\alpha_{1}+\alpha_{2}\right)-$ $\tau_{1}^{2} \lambda_{\max }\left(\beta_{3} R_{3}+\beta_{5} B_{2}^{T} P B_{2}\right)$. If (11) and LMI (12) hold, we have

$$
\mathscr{L} V\left(t, x_{t}\right) \leq-a\|x(t)\|^{2}+\left\|C^{T} P C\right\|\|r\|_{\infty}^{2} .
$$

Under an assumption that $V\left(t, x_{t}\right) \leq V\left(t_{0}, x_{t_{0}}\right)$ for all $t \geq t_{0}$, then

$$
\begin{aligned}
& \lambda_{\min }(P) \mathbb{E}\left\|x(t)+B_{2} \int_{t-\tau_{1}}^{t} x(s) d s\right\|^{2} \\
& \quad \leq V\left(t, x_{t}\right) \leq V\left(t_{0}, x_{t_{0}}\right) \leq \rho \mathbb{E}\|\varphi(\theta)\|^{2},
\end{aligned}
$$

where $\rho=\lambda_{\max }(P)+\tau_{1}\left(\left\|B_{2}^{T} P\right\|+\left\|P B_{2}\right\|\right)+\tau_{1}^{2}\left\|B_{2}^{T} P B_{2}\right\|^{2}+$ $\tau_{1} \beta_{1} \lambda_{\max }\left(R_{1}\right)+\tau_{1} \beta_{4} \alpha_{2}+\tau_{2} \beta_{2} \lambda_{\max }\left(R_{2}\right)+\left(\tau_{1}^{3} / 2\right) \lambda_{\max }\left(\beta_{3} R_{3}+\right.$ $\left.\beta_{5} B_{2}^{T} P B_{2}\right)$.

Thus, according to Theorem 1.3 in page 331 of [26], it can easily be gotten that

$$
\mathbb{E}\|x(t)\|^{2} \leq\left(\frac{1+\tau_{1}\left\|B_{2}\right\|}{1-\tau_{1}\left\|B_{2}\right\|}\right)^{2} \frac{\rho \mathbb{E}\|\varphi(\theta)\|^{2}}{\lambda_{\min }(P)} .
$$

If not, there exist $t>t_{0}$, such that $V(t, x(t)) \geq V(s, x(s))$ for all $s \in\left[t_{0}, t\right)$, and we get

$$
D^{+} \mathbb{E} V(t, x(t)) \geq 0 .
$$

In view of Ito's formula, we obtain

$$
D^{+} \mathbb{E} V(t, x(t))=\mathbb{E} \mathscr{L} V(t, x(t)) .
$$

By (24), (27), and (28), we get

$$
\begin{aligned}
0 & \leq D^{+} \mathbb{E} V(t, x(t))=\mathbb{E} \mathscr{L} V(t, x(t)) \\
& \leq-a \mathbb{E}\|x(t)\|^{2}+\left\|C^{T} P C\right\|\|r\|_{\infty}^{2} .
\end{aligned}
$$

So,

$$
\mathbb{E}\|x(t)\|^{2} \leq \frac{\left\|C^{T} P C\right\|}{a}\|r\|_{\infty}^{2} .
$$

By (26) and (30), we get

$$
\mathbb{E}\|x(t)\|^{2} \leq\left(\frac{1+\tau_{1}\left\|B_{2}\right\|}{1-\tau_{1}\left\|B_{2}\right\|}\right)^{2} \frac{\rho \mathbb{E}\|\varphi(\theta)\|^{2}}{\lambda_{\min }(P)}+\frac{\left\|C^{T} P C\right\|}{a}\|r\|_{\infty}^{2} .
$$


Thus

$$
\begin{aligned}
\mathbb{E}\|y(t)\|^{2} \leq & \|D\|^{2} \mathbb{E}\|x(t)\|^{2} \\
\leq & \left(\frac{1+\tau_{1}\left\|B_{2}\right\|}{1-\tau_{1}\left\|B_{2}\right\|}\right)^{2} \frac{\|D\|^{2} \rho \mathbb{E}\|\varphi(\theta)\|^{2}}{\lambda_{\min }(P)} \\
& +\frac{\|D\|^{2}\left\|C^{T} P C\right\|}{a}\|r\|_{\infty}^{2} \\
= & N_{1}+N_{2}\|r\|_{\infty}^{2},
\end{aligned}
$$

where

$$
\begin{aligned}
& N_{1}=\left(\frac{1+\tau_{1}\left\|B_{2}\right\|}{1-\tau_{1}\left\|B_{2}\right\|}\right)^{2} \frac{\|D\|^{2} \rho \mathbb{E}\|\varphi(\theta)\|^{2}}{\lambda_{\text {min }}(P)}, \\
& N_{2}=\frac{\|D\|^{2}\left\|C^{T} P C\right\|}{a} .
\end{aligned}
$$

By Definition 2, the nonlinear stochastic control system (1) is mean square BIBO stabilization. This completes the proof.

Theorem 7. For any given positive integer $\delta_{i}>0, i=1,2,3,4$, the nonlinear stochastic control system (1) with controller (3) is mean square BIBO stabilization, if there exist symmetric positive-definite matrices $P, Q$ and some positive constants $\eta$, $\delta_{5}$, and $\delta_{6}$ such that

$$
\begin{gathered}
B_{2} P=P B_{2}, \\
\delta_{1}-\delta_{2}-\delta_{3}>0 \\
\left(\delta_{1}-\delta_{2}-\delta_{3}\right) \lambda_{\min }(P)-\tau_{1}^{2} \lambda_{\max }\left(\delta_{4} P+\delta_{1} B_{2}^{T} P B_{2}\right) \\
-\lambda_{\max }\left(G^{T} P G\right)-\lambda_{\max }\left(Q^{-1}\right)-\lambda_{\max }\left(H^{T} P G Q G^{T} P H\right) \\
-\lambda_{\max }\left(H^{T} P H\right)-\delta_{5} \alpha^{2}>0,
\end{gathered}
$$

and the linear matrix inequality

$$
\Omega=\left(\begin{array}{cccccc}
\left(1_{*}, 1_{*}\right) & B_{1} S & 0 & \left(1_{*}, 4_{*}\right) & C & I \\
* & -\delta_{2} S & 0 & 0 & 0 & 0 \\
* & * & -\delta_{3} S & 0 & 0 & 0 \\
* & * & * & -\delta_{4} S & 0 & 0 \\
* & * & * & * & -\delta_{5} I & 0 \\
* & * & * & * & * & -\delta_{6} I
\end{array}\right) \leq 0
$$

holds, where

$$
\begin{gathered}
S=P^{-1} \\
\left(1_{*}, 1_{*}\right)=A S+S A^{T}+B_{2} S+S B_{2}^{T}-2 \eta C C^{T}+\delta_{1} S \\
\left(1_{*}, 4_{*}\right)=-A B_{2} S-B_{2} B_{2} S+\eta C C^{T} B_{2}-\delta_{1} B_{2} S .
\end{gathered}
$$

Proof. We define a Lyapunov functional $V\left(t, x_{t}\right)$ as

$$
V\left(t, x_{t}\right)=V_{1}\left(t, x_{t}\right)+V_{2}(t)+V_{3}(t)+V_{4}(t),
$$

where

$$
\begin{gathered}
V_{1}\left(t, x_{t}\right)=D^{T}\left(x_{t}\right) P D\left(x_{t}\right), \\
V_{2}(t)=\delta_{2} \int_{t-\tau_{1}}^{t} x^{T}(s) R_{1} x(s) d s,
\end{gathered}
$$

$V_{3}(t)=\int_{t-\tau_{2}}^{t} x^{T}(s)\left(\delta_{3} P+H^{T} P G Q G^{T} P H+H^{T} P H\right) x(s) d s$,

$$
V_{4}(t)=\tau_{1} \int_{t-\tau_{1}}^{t}\left(s-t+\tau_{1}\right) x^{T}(s)\left(\delta_{4} P+\delta_{1} B_{2}^{T} P B_{2}\right) x(s) d s .
$$

Taking the operator $\mathscr{L}$ of $V_{1}\left(t, x_{t}\right)$ along the trajectory of system (1), by (2), (34), and Definition 1, we have

$$
\begin{aligned}
\mathscr{L} V_{1}\left(t, x_{t}\right)= & D^{T}\left(x_{t}\right) P\left[\left(A+B_{2}+C K\right) x(t)\right. \\
& \left.+B_{1} x\left(t-\tau_{1}\right)+f(t, x(t))+C r(t)\right] \\
& +\left[\left(A+B_{2}+C K\right) x(t)\right. \\
& \left.\quad+B_{1} x\left(t-\tau_{1}\right)+C r(t)+f(t, x(t))\right]^{T} P D\left(x_{t}\right) \\
& +\frac{1}{2} \operatorname{trace}\left[\left(G x(t)+H x\left(t-\tau_{2}\right)\right)^{T}\right. \\
& \\
\leq & \xi(t)^{T} \widetilde{\Omega}^{2}(t)-\delta_{1} D^{T}\left(x_{t}\right) P D\left(x_{t}\right) \\
& +\delta_{2} x^{T}\left(t-\tau_{1}\right) P x\left(t-\tau_{1}\right) \\
& +\delta_{3} x^{T}\left(t-\tau_{2}\right) P x\left(t-\tau_{2}\right) \\
& +x\left(t-\tau_{2}\right) H^{T} P H x\left(t-\tau_{2}\right) \\
& +\delta_{1}\left(\int_{t-\tau_{1}}^{t} x(s) d s\right)^{T} B_{2}^{T} P D\left(x_{t}\right), \\
& \left.+\delta_{4}\left(\int_{t-\tau_{1}}^{t} x(s) d s\right)^{T} P\left(\int_{t-\tau_{1}}^{t} x(s) d s\right)\right] \\
& +\delta_{5} \alpha^{2}\|x(t)\|^{2}+\beta_{6}\|r\|_{\infty}^{2} \\
& +x\left(t-\tau_{2}\right) H^{T} P G x(t) \\
& +x^{T}(t) G^{T} P G x(t)+x^{T}(t) G^{T} P H x\left(t-\tau_{2}\right) \\
& \\
&
\end{aligned}
$$


where

$$
\begin{gathered}
\xi(t)^{T}=\left[D^{T}\left(x_{t}\right), x^{T}\left(t-\tau_{1}\right), x^{T}\left(t-\tau_{2}\right),\right. \\
\left.\left(\int_{t-\tau_{1}}^{t} x(s) d s\right)^{T}, f^{T}(x, x(t)), r^{T}(t)\right], \\
\widetilde{\Omega}=\left(\begin{array}{cccccc}
\left(1^{*}, 1^{*}\right) & \delta_{1} P & 0 & \left(1^{*}, 4^{*}\right) & P C & P \\
* & -\delta_{2} P & 0 & 0 & 0 & 0 \\
* & * & -\delta_{3} P & 0 & 0 & 0 \\
* & * & * & -\delta_{4} P & 0 & 0 \\
* & * & * & * & -\delta_{5} I & 0 \\
* & * & * & * & * & -\delta_{6} I
\end{array}\right),
\end{gathered}
$$

with

$$
\begin{gathered}
\left(1^{*}, 1^{*}\right)=P\left(A+B_{2}\right)+\left(A+B_{2}\right)^{T} P+P B_{1}-2 \eta P C^{T} C P, \\
\left(1^{*}, 4^{*}\right)=-P A B_{2}-P B_{2}^{2}+\eta P C C^{T} B_{2} P-\delta_{1} P B_{2} .
\end{gathered}
$$

Pre- and postmultiplying (36) by $\operatorname{diag}[P, P, P, P, I, I]$, we can obtain

$$
\widetilde{\Omega} \leq 0
$$

Then, by Lemmas 4 and 5, (40), and (43), we conclude that

$$
\begin{aligned}
\mathscr{L} V_{1}\left(t, x_{t}\right) \leq & -\delta_{1} x^{T}(t) P x(t)+\delta_{2} x^{T}\left(t-\tau_{1}\right) P x\left(t-\tau_{1}\right) \\
& +\delta_{6}\|r\|_{\infty}^{2}+\delta_{3} x^{T}\left(t-\tau_{2}\right) P x\left(t-\tau_{2}\right) \\
& +x^{T}(t) G^{T} P G x(t) \\
& +\tau_{1} \int_{t-\tau_{1}}^{t} x^{T}(s)\left(\delta_{4} P+\delta_{1} B_{2}^{T} P B_{2}\right) x(s) d s \\
& +x^{T}\left(t-\tau_{2}\right) H^{T} P G Q G^{T} P H x\left(t-\tau_{2}\right) \\
& +x^{T}(t) Q^{-1} x(t) \\
& +x^{T}\left(t-\tau_{2}\right) H^{T} P H x\left(t-\tau_{2}\right)+\delta_{5} \alpha^{2}\|x(t)\|^{2} .
\end{aligned}
$$

Taking the operator $\mathscr{L}$ of $V_{i}(t), i=2,3,4$, along the trajectory of system (1), we get

$$
\begin{aligned}
\mathscr{L} V_{2}(t) & =\delta_{2} x^{T}(t) P x(t)-\delta_{2} x^{T}\left(t-\tau_{1}\right) P x\left(t-\tau_{1}\right), \\
\mathscr{L} V_{3}(t)= & x^{T}(t)\left(\delta_{3} P+H^{T} P G Q G^{T} P H+H^{T} P H\right) x(t) \\
& -x^{T}\left(t-\tau_{2}\right)\left(\delta_{3} P+H^{T} P G Q G^{T} P H+H^{T} P H\right) \\
& \times x\left(t-\tau_{2}\right),
\end{aligned}
$$$$
\mathscr{L} V_{4}(t)=\tau_{1}^{2} x^{T}(t)\left(\delta_{4} P+\delta_{1} B_{2}^{T} P B_{2}\right) x(t)
$$$$
-\tau_{1} \int_{t-\tau_{1}}^{t} x^{T}(s)\left(\delta_{4} P+\delta_{1} B_{2}^{T} P B_{2}\right) x(s) d s .
$$

Combining (44) with (46), we have

$$
\begin{aligned}
\mathscr{L} V(t, x(t)) \leq & -\left(\delta_{1}-\delta_{2}-\delta_{3}\right) x^{T}(t) P x(t) \\
& +\beta_{5} \alpha^{2}\|x(t)\|^{2}+\beta_{6}\|r\|_{\infty}^{2} \\
& +\tau_{1}^{2} x^{T}(t)\left(\delta_{4} P+\delta_{1} B_{2}^{T} P B_{2}\right) x(t) \\
& +x^{T}(t) Q^{-1} x(t) \\
& +x^{T}(t) H^{T} P H x(t)+x^{T}(t) G^{T} P G x(t) \\
& +x^{T}(t) H^{T} P G Q G^{T} P H x(t) \\
\leq & -\left[\left(\delta_{1}-\delta_{2}-\delta_{3}\right) \lambda_{\min }(P)\right. \\
& \quad-\tau_{1}^{2} \lambda_{\max }\left(\delta_{4} P+\delta_{1} B_{2}^{T} P B_{2}\right) \\
& \quad-\beta_{5} \alpha^{2}-\lambda_{\max }\left(H^{T} P H\right)-\lambda_{\max }\left(G^{T} P G\right) \\
& \left.\quad-\lambda_{\max }\left(Q^{-1}\right)-\lambda_{\max }\left(H^{T} P G Q G^{T} P H\right)\right] \\
\times & \|x(t)\|^{2}+\beta_{6}\|r\|_{\infty}^{2} .
\end{aligned}
$$

Let $b=\left(\delta_{1}-\delta_{2}-\delta_{3}\right) \lambda_{\text {min }}(P)-\tau_{1}^{2} \lambda_{\text {max }}\left(\delta_{4} P+\delta_{1} B_{2}^{T} P B_{2}\right)-$ $\beta_{5} \alpha^{2}-\lambda_{\max }\left(H^{T} P H\right)-\lambda_{\max }\left(G^{T} P G\right)-\lambda_{\max }\left(Q^{-1}\right)-$ $\lambda_{\max }\left(H^{T} P G Q G^{T} P H\right)$; we have

$$
\mathscr{L} V(t, x(t)) \leq-b\|x(t)\|^{2}+\beta_{6}\|r\|_{\infty}^{2} .
$$

The rest of the proof is essentially the same as Theorem 6 and hence is omitted. This completes the proof.

If the stochastic term disappears, the control system (9) reduces to

$$
\begin{gathered}
d\left[x(t)+B_{2} \int_{t-\tau_{1}}^{t} x(s) d s\right] \\
=\left[\left(A+B_{2}+C K\right) x(t)+B_{1} x\left(t-\tau_{1}\right)+C r(t)\right. \\
\left.\quad+f\left(t, x(t), x\left(t-\tau_{1}\right)\right)\right] d t \quad t \geq t_{0} \geq 0, \\
y(t)=D x(t), \\
x(\theta)=\varphi(\theta) \in C_{\mathscr{F}_{0}}^{b}\left(\left[t_{0}-\tau, t_{0}\right] ; R^{n}\right), \quad \theta \in\left[t_{0}-\tau, t_{0}\right] .
\end{gathered}
$$

We have the following stabilization results.

Corollary 8. For any given positive constants $\beta_{i}>0, i=$ $1,2,3,4,5$, the nonlinear stochastic control system (1) with the controller (3) is BIBO stabilization, if there exist symmetric positive-definite matrices $P, R_{1}, R_{2}, R_{3}$, and $X$, such that

$$
\begin{aligned}
& \beta_{5} \lambda_{\text {min }}(P)-\beta_{1} \lambda_{\text {max }}\left(R_{1}\right)-\beta_{2} \lambda_{\text {max }}\left(R_{2}\right) \\
& \quad-\beta_{4}\left(\alpha_{1}+\alpha_{2}\right)-\tau_{1}^{2} \lambda_{\max }\left(\beta_{3} R_{3}+\beta_{5} B_{2}^{T} P B_{2}\right)>0,
\end{aligned}
$$


and the linear matrix inequality

$$
\widetilde{\Xi}=\left(\begin{array}{ccccc}
(1,1) & P B_{1} & 0 & (1,4) & P \\
* & (2,2) & 0 & 0 & 0 \\
* & * & (3,3) & 0 & 0 \\
* & * & * & (4,4) & 0 \\
* & * & * & * & -\beta_{4} I
\end{array}\right) \leq 0
$$

with

$$
\begin{gathered}
(1,1)=P A+A^{T} P+P B_{2}+B_{2}^{T} P-X-X^{T}+\beta_{5} P+P, \\
(1,4)=-P A B_{2}-P B_{2}^{2}+X B_{2}-\beta_{5} P B_{2}, \\
(2,2)=-\beta_{1} R_{1} \\
(3,3)=-\beta_{2} R_{2} \\
(4,4)=-\beta_{3} R_{3} .
\end{gathered}
$$

Corollary 9. For any given positive integer $\delta_{i}>0, i=1,2,3$, the nonlinear stochastic control system (49) with the controller (3) is BIBO stabilization, if there exist symmetric positivedefinite matrix $P>0$ and some positive constants $\eta>0$, $\delta_{4}>0$, and $\delta_{5}>0$ such that

$$
\begin{gathered}
B_{2} P=P B_{2} \\
\delta_{1}-\delta_{2}>0 \\
\left(\delta_{1}-\delta_{2}\right) \lambda_{\min }(P)-\tau_{1}^{2} \lambda_{\max }\left(\delta_{3} P+\delta_{1} B_{2}^{T} P B_{2}\right)-\delta_{5} \alpha^{2}>0
\end{gathered}
$$

and the linear matrix inequality

$$
\Omega=\left(\begin{array}{ccccc}
\left(1_{*}, 1_{*}\right) & B_{1} S & \left(1_{*}, 3_{*}\right) & C & I \\
* & -\delta_{2} S & 0 & 0 & 0 \\
* & * & -\delta_{3} S & 0 & 0 \\
* & * & * & -\delta_{4} I & 0 \\
* & * & * & * & -\delta_{5} I
\end{array}\right) \leq 0
$$

holds, where

$$
\begin{gathered}
S=P^{-1} \\
\left(1_{*}, 1_{*}\right)=A S+S A^{T}+B_{2} S+S B_{2}^{T}-2 \eta C C^{T}+\delta_{1} S \\
\left(1_{*}, 3_{*}\right)=-A B_{2} S-B_{2}^{2} S+\eta C C^{T} B_{2}-\delta_{1} B_{2} S .
\end{gathered}
$$

\section{Examples}

In this section, Example 1 will be presented to show that the mean BIBO stabilization conditions in Theorem 6 are valid, Example 2 will be presented to show that the BIBO stabilization conditions in Corollary 8 are valid, and Example 3 will be presented to show that the derived conditions are much less conservative than those given in the literature [20].
Example 1. As a simple application of Theorem 6, consider the stochastic control system (1) with control law (3); the parameters are given by

$$
\begin{aligned}
& A=\left(\begin{array}{cc}
-2 & 1 \\
1 & -3
\end{array}\right), \quad B=\left(\begin{array}{cc}
-2 & 0 \\
0 & -1
\end{array}\right), \\
& C=\left(\begin{array}{ll}
4 & 1 \\
2 & 0
\end{array}\right), \quad G=\left(\begin{array}{cc}
0.05 & 0 \\
0 & 0.05
\end{array}\right),
\end{aligned}
$$

$$
H=\left(\begin{array}{cc}
0.01 & 0 \\
0 & 0.01
\end{array}\right)
$$

$f_{1}=[0.1 \sin (t), 0.1 \cos (t)]^{T}, f_{2}=[\sqrt{0.2} \sin (t), \sqrt{0.2} \cos (t)]^{T}$, $\beta_{1}=0.01, \beta_{2}=0.01, \beta_{3}=0.01, \beta_{4}=1, \beta_{5}=4.8, \alpha_{1}=0.01$, and $\alpha_{2}=0.02$. Let us decompose matrix $B=B_{1}+B_{2}$, where

$$
B_{1}=\left(\begin{array}{cc}
-0.5 & 0.1 \\
0.2 & -0.5
\end{array}\right), \quad B_{2}=\left(\begin{array}{cc}
-1.5 & -0.1 \\
-0.2 & -0.5
\end{array}\right) \text {. }
$$

By using the Matlab LMI Toolbox, we solve LMI (12) and obtain the feasible solutions as follows:

$$
\begin{gathered}
P=\left(\begin{array}{ll}
431.2920 & 131.9598 \\
131.9598 & 408.9760
\end{array}\right), \quad R_{1}=\left(\begin{array}{cc}
5500.9 & -492.4 \\
-492.4 & 5151.3
\end{array}\right), \\
R_{2}=\left(\begin{array}{cc}
3183.9 & 0.7 \\
0.7 & 3183.7
\end{array}\right), \quad R_{3}=\left(\begin{array}{cc}
3423.6 & -251 \\
-251 & 3991.8
\end{array}\right), \\
X=\left(\begin{array}{ll}
695.8768 & 469.2695 \\
469.2695 & 801.3229
\end{array}\right), \\
\beta_{5} \lambda_{\min }(P)-\beta_{1} \lambda_{\max }\left(R_{1}\right)-\beta_{2} \lambda_{\max }\left(R_{2}\right)-\beta_{4}\left(\alpha_{1}+\alpha_{2}\right) \\
-\tau_{1}^{2} \lambda_{\max }\left(\beta_{3} R_{3}+\beta_{5} B_{2}^{T} P B_{2}\right)=2.0586>0 .
\end{gathered}
$$

From the above formula, we can see that condition (11) is met. The stabilizing feedback gain matrix is given by

$$
K=-C^{-1} P^{-1} X=\left(\begin{array}{cc}
-0.3477 & -0.8922 \\
-0.0097 & 3.0268
\end{array}\right) \text {. }
$$

Meanwhile, we obtain the maximum value $\tau_{\max }=5.315$. This example shows that the mean square BIBO stabilization conditions in Theorem 6 are valid.

Example 2. Let us consider the delayed control system (49) with parameters given by

$$
\begin{gathered}
A=\left(\begin{array}{cc}
-4 & 1 \\
1 & -4
\end{array}\right), \quad B=\left(\begin{array}{cc}
-2 & 0 \\
0 & -1
\end{array}\right), \\
C=\left(\begin{array}{ll}
2 & 1 \\
1 & 0
\end{array}\right),
\end{gathered}
$$

$\beta_{1}=0.01, \beta_{2}=0.01, \beta_{3}=0.01, \beta_{4}=1, \beta_{5}=4.8, \alpha_{1}=0.01$, and $\alpha_{2}=0.02$.

Let us decompose matrix $B=B_{1}+B_{2}$, where

$$
B_{1}=\left(\begin{array}{cc}
-0.5 & 0.1 \\
0.2 & -0.5
\end{array}\right), \quad B_{2}=\left(\begin{array}{cc}
-1.5 & -0.1 \\
-0.2 & -0.5
\end{array}\right) \text {. }
$$


Now we use Corollary 8 in this paper to study the problem. By using the Matlab LMI Toolbox, we obtain the feasible solutions as follows:

$$
\begin{gathered}
P=\left(\begin{array}{ll}
1.8869 & 1.7503 \\
1.7503 & 3.1049
\end{array}\right), \quad R_{1}=\left(\begin{array}{cc}
21.0832 & 1.9140 \\
1.9140 & 24.2362
\end{array}\right) \\
R_{2}=\left(\begin{array}{cc}
18.6240 & 0 \\
0 & 18.6240
\end{array}\right), \quad R_{3}=\left(\begin{array}{cc}
21.7687 & -5.7602 \\
-5.7602 & 25.2583
\end{array}\right) \\
X=\left(\begin{array}{ll}
0.5918 & 1.0013 \\
1.0013 & 4.1015
\end{array}\right) \\
\beta_{5} \lambda_{\min }(P)-\beta_{1} \lambda_{\max }\left(R_{1}\right)-\beta_{2} \lambda_{\max }\left(R_{2}\right)-\beta_{4}\left(\alpha_{1}+\alpha_{2}\right) \\
-\tau_{1}^{2} \lambda_{\max }\left(\beta_{3} R_{3}+\beta_{5} B_{2}^{T} P B_{2}\right)=0.0043>0
\end{gathered}
$$

From the above formula, we can see that condition (50) is met. The stabilizing feedback gain matrix is given by

$$
K=-C^{-1} P^{-1} X=\left(\begin{array}{cc}
-0.3054 & -2.1417 \\
0.5804 & 5.7392
\end{array}\right) \text {. }
$$

Meanwhile, we obtain the maximum value $\tau_{\max }=0.48$.

Example 3. For the convenience of comparison, let us consider a delayed control system (49) with parameters given by

$$
A=\left(\begin{array}{cc}
-4 & 1 \\
1 & -4
\end{array}\right), \quad B=\left(\begin{array}{cc}
-2 & 0 \\
0 & -1
\end{array}\right), \quad C=\left(\begin{array}{ll}
2 & 1 \\
1 & 0
\end{array}\right),
$$

$\delta_{1}=0.8, \delta_{2}=0.4$, and $\delta_{3}=0.5$.

Now we use Corollary 9 in this paper to study the problem; let us decompose matrix $B=B_{1}+B_{2}$, where

$$
B_{1}=\left(\begin{array}{cc}
-1.95 & 0 \\
0 & -1.03
\end{array}\right), \quad B_{2}=\left(\begin{array}{cc}
-0.05 & 0 \\
0 & 0.03
\end{array}\right) \text {. }
$$

By using the Matlab LMI Toolbox, solving LMI (55), we can get

$$
P=\left(\begin{array}{cc}
14.8464 & 0.8740 \\
0.8740 & 6.1426
\end{array}\right)
$$

and $\delta_{4}=1.0428, \delta_{5}=1.0428$, and $\eta=0.0683$. In order to verify condition (54), we give the parameters as follows:

$$
\begin{aligned}
& \left(\delta_{1}-\delta_{2}\right) \lambda_{\min }(P)-\tau_{1}^{2} \lambda_{\max }\left(\delta_{3} P+\delta_{1} B_{2}^{T} P B_{2}\right) \\
& -\delta_{5} \alpha^{2}=0.0145>0 .
\end{aligned}
$$

From the above formula, we can see that condition (54) is met. The stabilizing feedback gain matrix is given by

$$
K=\left(\begin{array}{ll}
-2.0867 & -0.5387 \\
-1.0135 & -0.0597
\end{array}\right)
$$

Meanwhile, solving LMI (55), the maximum value of $\tau_{\max }$ for BIBO stabilization of system (49) is $\tau_{\max }=1.06$. In [20], its $\tau_{\max }$ is 0.2960 . The maximum value of $\tau_{\max }$ in this example in our paper is $358.11 \%$ larger than this in [20]. This example shows that the BIBO stabilization conditions in this paper are less conservative than these in [20]. The essential reasons why the stability conditions we have given are less conservative are that we decompose the coefficient matrix in a proper way.

\section{Conclusions}

The problem of the mean square BIBO stabilization for the stochastic control systems with delays and nonlinear perturbations is investigated. A class of suitable Lyapunov functional combined with the descriptor model transformation and the decomposition technique of coefficient matrix is constructed to derive some novel delay-dependent BIBO stabilization criteria. Numerical examples have shown that the derived conditions are valid and improvements over the existing results are significant.

\section{Acknowledgments}

The authors would like to thank the editor and the reviewers for their detailed comments and valuable suggestions which have led to a much improved paper. The work of Xia Zhou is supported by the National Natural Science Foundation of China (no. 11226140), the Anhui Provincial Colleges and Universities Natural Science Foundation (no. KJ2013Z267), and Fuyang Teachers College Natural Science Foundation (no. 2012FSKJ08). The work of Shouming Zhong is supported by National Basic Research Program of China (no. 2010CB732501).

\section{References}

[1] K. Ramakrishnan and G. Ray, "Robust stability criteria for a class of uncertain discrete-time systems with time-varying delay," Applied Mathematical Modelling, vol. 37, no. 3, pp. 14681479, 2013.

[2] R. F. Rao, X. R. Wang, S. M. Zhong, and Z. L. Pu, "LMI approach to exponential stability and almost sure exponential stability for stochastic fuzzy Markovian-Jumping Cohen-Grossberg neural networks with nonlinear $p$-laplace diffusion," Journal of Applied Mathematics, vol. 2013, Article ID 396903, 21 pages, 2013.

[3] Q.-L. Han, "A descriptor system approach to robust stability of uncertain neutral systems with discrete and distributed delays," Automatica, vol. 40, no. 10, pp. 1791-1796, 2004.

[4] S. Long and $\mathrm{D}$. Xu, "Global exponential stability of nonautonomous cellular neural networks with impulses and timevarying delays," Communications in Nonlinear Science and Numerical Simulation, vol. 18, no. 6, pp. 1463-1472, 2013.

[5] Z. X. Liu, S. Lv, and S. M. Zhong, "Augmented Lyapunov method for BIBO stabilization of discrete system," Journal of Mathematics Research, vol. 2, pp. 116-122, 2010.

[6] S. M. Zhong and Y. Q. Huang, "Bounded input and bounded output for nonlinear systems with time-delay," Journal of University of Electronic Science and Technology of China, vol. 29, no. 6, pp. 655-657, 2000 (Chinese).

[7] K. C. Cao, S. M. Zhong, and B. S. Liu, "BIBO and robust stabilization for system with time-delay and nonlinear perturbations," Journal of University of Electronic Science and Technology of China, vol. 32, pp. 787-789, 2003 (Chinese).

[8] K. A. Moornani and M. Haeri, "Necessary and sufficient conditions for BIBO-stability of some fractional delay systems of neutral type," IEEE Transactions on Automatic Control, vol. 56, no. 1, pp. 125-128, 2011.

[9] E. Awwad, I. Gyori, and F. Hartung, "BIBO stabilization of feedback control systems with time dependent delays," Applied 
Mathematics and Computation, vol. 219, no. 8, pp. 3664-3676, 2012.

[10] J. R. Partington and C. Bonnet, " $L_{\infty}$ and BIBO stabilization of delay systems of neutral type," Systems \& Control Letters, vol. 52, no. 3-4, pp. 283-288, 2004.

[11] K. H. You and E. B. Lee, "BIBO stability integral ( $L_{\infty}$-gain) for second-order systems with numerator dynamics," Automatica, vol. 36, no. 11, pp. 1693-1699, 2000.

[12] A. T. Tomerlin and W. W. Edmonson, "BIBO stability of d-dimensional filters," Multidimensional Systems and Signal Processing, vol. 13, no. 3, pp. 333-340, 2002.

[13] W. Wang and Y. Zou, "The stabilizability and connections between internal and BIBO stability of 2-D singular systems," Multidimensional Systems and Signal Processing, vol. 15, no. 1, pp. 37-50, 2004.

[14] L.-L. Xiong, S.-M. Zhong, and M. Ye, "Delay-dependent BIBO stability analysis of switched uncertain neutral systems," Mathematical and Computer Modelling, vol. 53, no. 9-10, pp. 16071620, 2011.

[15] B. Wang, J. Wang, X. Liu, and S. M. Zhong, "New results on BIBO stability analysis for a class of neutral delay systems," Journal of the Franklin Institute, vol. 348, no. 2, pp. 426-437, 2011.

[16] J. Wei, P. Shi, H. R. Karimi, and B. Wang, "BIBO stability analysis for delay switched systems with nonlinear perturbation," Abstract and Applied Analysis, vol. 2013, Article ID 738653, 8 pages, 2013.

[17] P. Li and S.-M. Zhong, "BIBO stabilization of time-delayed system with nonlinear perturbation," Applied Mathematics and Computation, vol. 195, no. 1, pp. 264-269, 2008.

[18] P. Li and S.-M. Zhong, "BIBO stabilization for system with multiple mixed delays and nonlinear perturbations," Applied Mathematics and Computation, vol. 196, no. 1, pp. 207-213, 2008.

[19] P. Li and S.-M. Zhong, "BIBO stabilization of piecewise switched linear systems with delays and nonlinear perturbations," Applied Mathematics and Computation, vol. 213, no. 2, pp. 405-410, 2009.

[20] P. Li, S.-M. Zhong, and J.-Z. Cui, "Delay-dependent robust BIBO stabilization of uncertain system via LMI approach," Chaos, Solitons and Fractals, vol. 40, no. 2, pp. 1021-1028, 2009.

[21] Y. Fu and X. Liao, "BIBO stabilization of stochastic delay systems with uncertainty," IEEE Transactions on Automatic Control, vol. 48, no. 1, pp. 133-138, 2003.

[22] X. Zhou and S. Zhong, "Riccati equations and delay-dependent BIBO stabilization of stochastic systems with mixed delays and nonlinear perturbations," Advances in Difference Equations, vol. 2010, Article ID 494607, 14 pages, 2010.

[23] K. Gu, "An integral inequality in the stability problem of timedelay systems," in Proceedings of the 39th IEEE Confernce on Decision and Control, IEEE Control Systems Society, pp. 28052810, IEEE Publisher, Sydney, Australia, December 2000.

[24] Z. Wang, H. Shu, Y. Liu, D. W. C. Ho, and X. Liu, "Robust stability analysis of generalized neural networks with discrete and distributed time delays," Chaos, Solitons \& Fractals, vol. 30, no. 4, pp. 886-896, 2006.

[25] J. Hale, Theory of Functional Differential Equation, Springer, New York, NY, USA, 1977.

[26] V. Kolmanovskii and A. Myshkis, Introduction to the Theory and Applications of Functional Differential Equations, vol. 463, Kluwer Academic Publishers, Dordrecht, The Netherlands, 1999. 


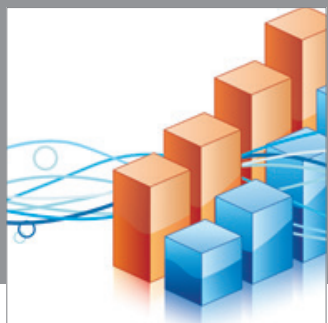

Advances in

Operations Research

mansans

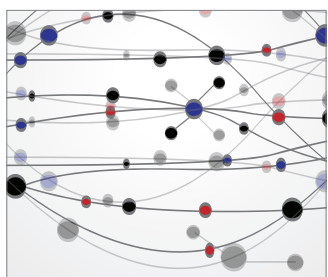

The Scientific World Journal
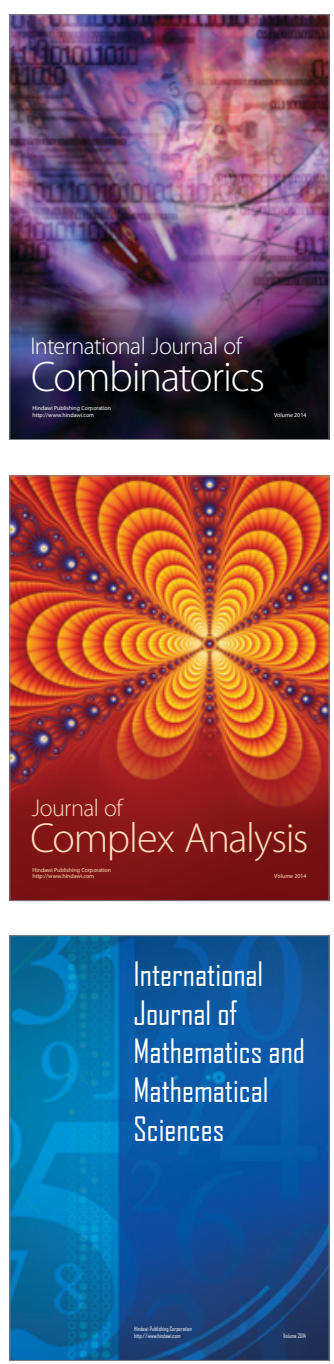
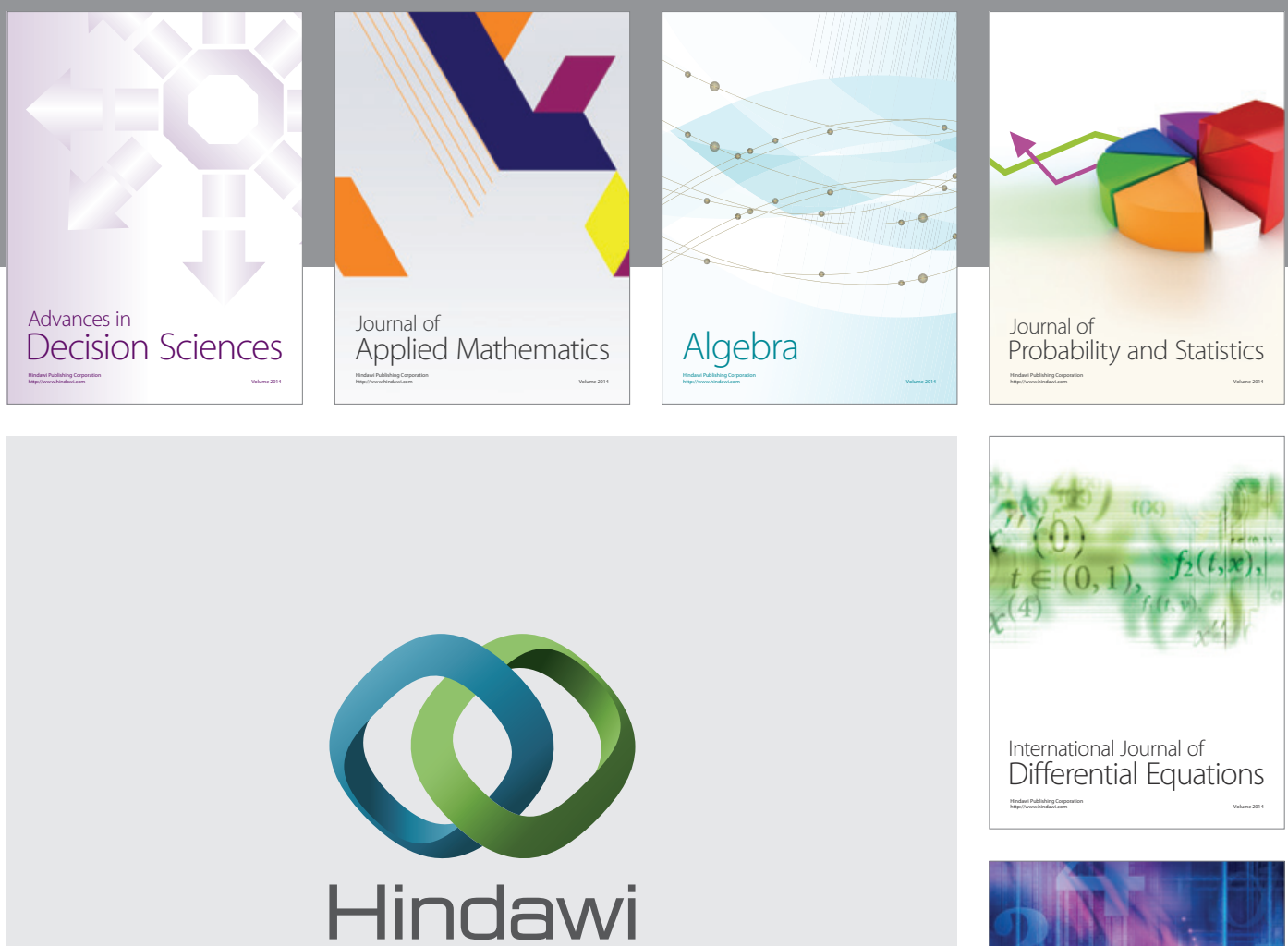

Submit your manuscripts at http://www.hindawi.com
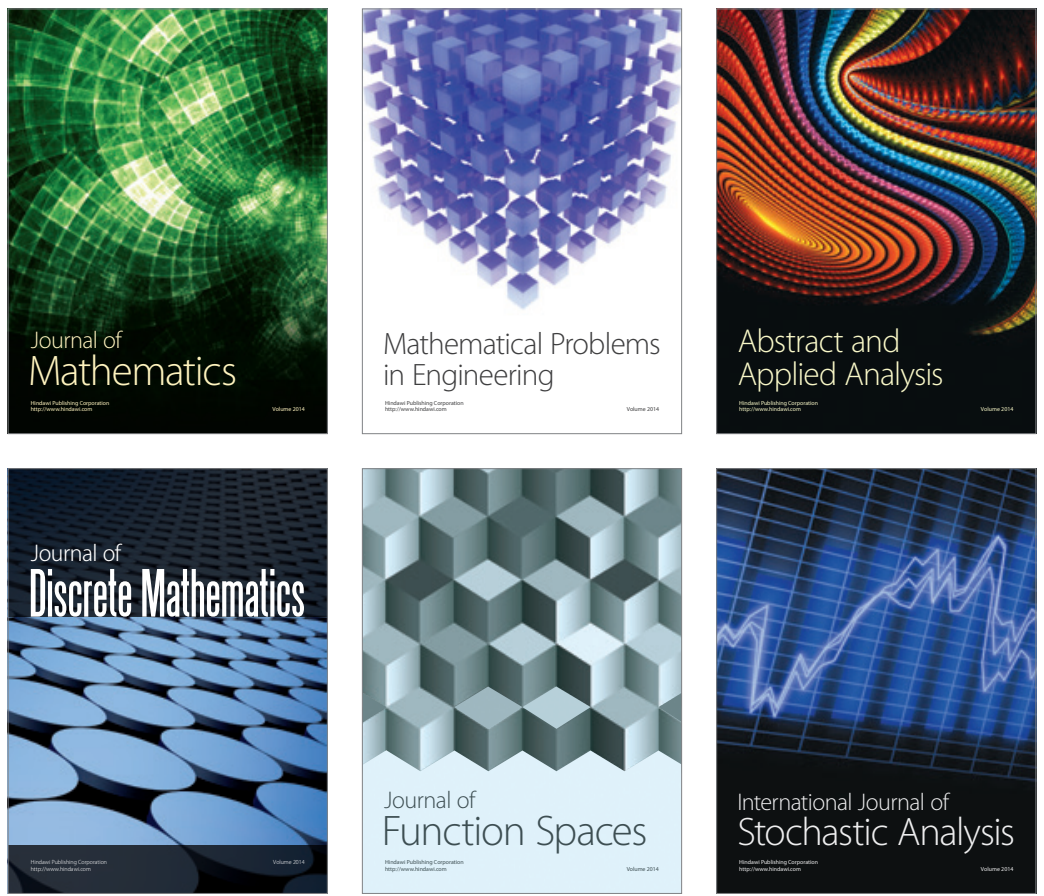

Journal of

Function Spaces

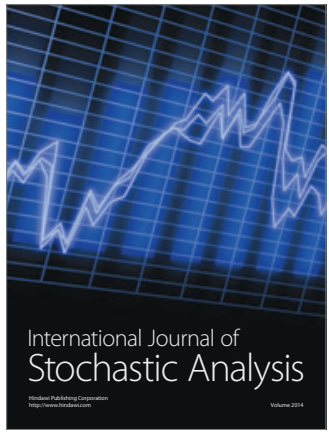

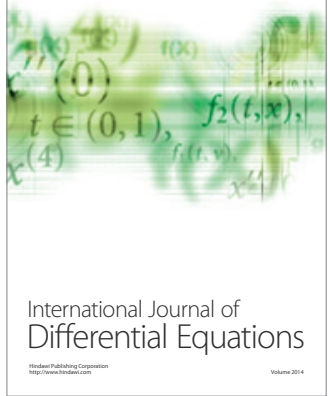
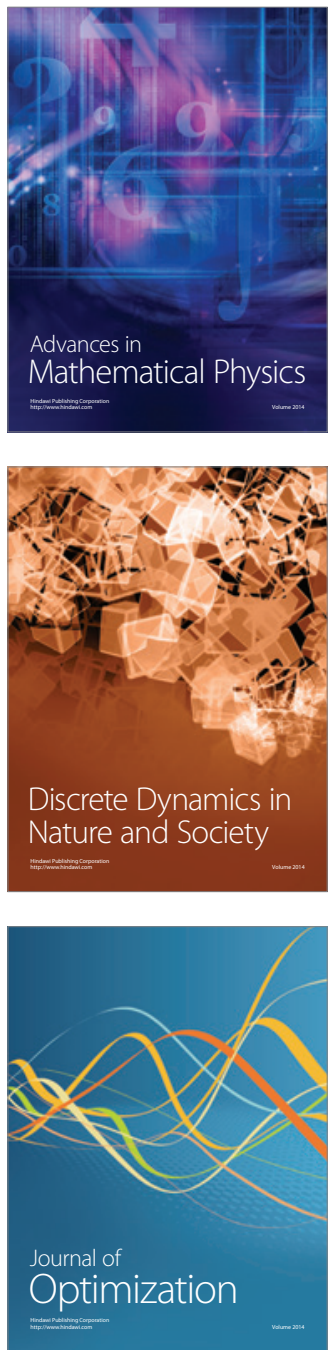